\title{
INDEX
}

\section{Volume 14, Nos. 1 and 2, 1972}

All samples published in Volume 14, Nos. 1 and 2, 1972 are listed here in index form. They appear alphabetically, by laboratory, and in numerical order. Page numbers for each sample are indicated.

\begin{tabular}{|c|c|c|c|c|c|c|c|}
\hline $\begin{array}{c}\text { Sample } \\
\text { no. }\end{array}$ & $\begin{array}{c}\text { Page } \\
\text { no. }\end{array}$ & $\begin{array}{c}\text { Sample } \\
\text { no. }\end{array}$ & $\begin{array}{c}\text { Page } \\
\text { no. }\end{array}$ & $\begin{array}{c}\text { Sample } \\
\text { no. }\end{array}$ & $\begin{array}{c}\text { Page } \\
\text { no. }\end{array}$ & $\begin{array}{c}\text { Sample } \\
\text { no. }\end{array}$ & $\begin{array}{c}\text { Page } \\
\text { no. }\end{array}$ \\
\hline AERIK & & ALG & & Gif & & Gif & \\
\hline-8 & 274 & -28 & 1 & -890 & 291 & -1153 & 302 \\
\hline-9 & 276 & -29 & 2 & -1007 & 284 & -1154 & 302 \\
\hline-10 & 276 & -30 & 5 & -1008 & 284 & -1155 & 302 \\
\hline-11 & 276 & -31 & 2 & -1033 & 299 & -1156 & 285 \\
\hline-12 & 276 & -32 & 2 & -1034 & 299 & -1157 & 286 \\
\hline-13 & 276 & -33 & 2 & -1035 & 299 & -1158 & 289 \\
\hline-14 & 276 & -34 & 2 & -1036 & 299 & -1159 & 288 \\
\hline-15 & 276 & -35 & 2 & -1037 & 299 & -1160 & 288 \\
\hline-16 & 276 & -36 & 2 & -1038 & 299 & -1161 & 285 \\
\hline-17 & 276 & -37 & 5 & -1039 & 299 & -1162 & 307 \\
\hline-18 & 277 & -38 & 3 & -1040 & 300 & -1163 & 307 \\
\hline-19 & 277 & -39 & 3 & -1041 & 300 & -1164 & 306 \\
\hline-20 & 277 & -40 & 3 & -1042 & 299 & -1167 & 300 \\
\hline-21 & 277 & -41 & 3 & -1043 & 300 & -1169 & 302 \\
\hline-22 & 278 & -42 & 3 & -1048 & 298 & -1171 & 294 \\
\hline-23 & 278 & -43 & 4 & -1049 & 298 & -1172 & 294 \\
\hline-24 & 278 & -44 & 4 & -1050 & 298 & -1181 & 294 \\
\hline-25 & 278 & -46 & 4 & -1051 & 299 & -1182 & 294 \\
\hline-26 & 278 & -47 & 4 & -1052 & 298 & -1183 & 294 \\
\hline-27 & 278 & -48 & 4 & -1057 & 301 & -1184 & 303 \\
\hline-28 & 278 & -49 & 3 & -1080 & 308 & -1186 & 281 \\
\hline$-2,9$ & 278 & -50 & 5 & -1081 & 297 & -1187 & 281 \\
\hline-30 & 278 & -51 & 5 & -1082 & 297 & -1188 & 282 \\
\hline-31 & 279 & & & -1083 & 297 & -1189 & 282 \\
\hline .32 & 279 & Gif & & -1086 & 288 & -1190 & 282 \\
\hline-33 & 279 & -355 & 290 & -1087 & 288 & -1191 & 306 \\
\hline-34 & 279 & -356 & 290 & -1088 & 284 & -1192 & 315 \\
\hline-35 & 279 & -568 & 313 & -1090 & 290 & -1193 & 315 \\
\hline-36 & 279 & -569 & 313 & -1091 & 285 & -1195 & 293 \\
\hline-37 & 279 & -574 & 313 & -1092 & 285 & -1197 & 307 \\
\hline-38 & 279 & -575 & 313 & -1093 & 285 & -1198 & 307 \\
\hline-39 & 279 & -576 & 313 & -1106 & 290 & -1199 & 308 \\
\hline-40 & 279 & -577 & 313 & -1107 & 290 & -1200 & 308 \\
\hline-41 & 279 & -578 & 313 & -1108 & 291 & -1201 & 286 \\
\hline-42 & 279 & -740 & 294 & -1109 & 290 & -1202 & 286 \\
\hline-43 & 279 & -741 & 294 & -1111 & 287 & -1203 & 287 \\
\hline-44 & 279 & -742 & 294 & -1112 & 283 & -1204 & 287 \\
\hline-45 & 279 & -865 & 308 & -1120 & 291 & -1205 & 298 \\
\hline-46 & 279 & -866 & 304 & -1123 & 317 & -1206 & 298 \\
\hline-47 & 279 & -872 & 301 & -1124 & 317 & -1207 & 309 \\
\hline-48 & 279 & -873 & 300 & -1125 & 317 & -1208 & 309 \\
\hline-49 & 279 & -879 & 293 & -1126 & 293 & -1209 & 309 \\
\hline-50 & 279 & -880 & 293 & -1128 & 284 & -1210 & 309 \\
\hline-51 & 279 & -881 & 291 & -1131 & 318 & -1211 & 309 \\
\hline-52 & 279 & -882 & 291 & -1132 & 318 & -1212 & 309 \\
\hline-53 & 279 & -883 & 291 & -1133 & 318 & -1214 & 303 \\
\hline & & -884 & 292 & -1134 & 318 & -1216 & 303 \\
\hline ALG & & -885 & 292 & -1135 & 307 & -1217 & 303 \\
\hline-5 & 4 & -886 & 292 & -1138 & 309 & -1218 & 303 \\
\hline-25 & 1 & -887 & 292 & -1139 & 309 & -1219 & 303 \\
\hline-26 & 1 & -888 & 292 & -1149 & 282 & -1220 & 292 \\
\hline-27 & 1 & -889 & 292 & -1152 & 301 & -1221 & 292 \\
\hline
\end{tabular}


Index

\begin{tabular}{|c|c|c|c|c|c|c|c|}
\hline $\begin{array}{c}\text { Sample } \\
\text { no. }\end{array}$ & $\begin{array}{c}\text { Page } \\
\text { no. }\end{array}$ & $\begin{array}{c}\text { Sample } \\
\text { no. }\end{array}$ & $\begin{array}{c}\text { Page } \\
\text { no. }\end{array}$ & $\begin{array}{c}\text { Sample } \\
\text { no. }\end{array}$ & $\begin{array}{c}\text { Page } \\
\text { no. }\end{array}$ & $\begin{array}{c}\text { Sample } \\
\text { no. }\end{array}$ & $\begin{array}{c}\text { Page } \\
\text { no. }\end{array}$ \\
\hline Gif & & Gif & & Gif & & Gif & \\
\hline-1222 & 293 & -1330 & 283 & -1489 & 286 & -1806 & 315 \\
\hline-1224 & 288 & -1331 & 287 & -1490 & 286 & -1822 & 296 \\
\hline-1225 & 289 & -1332 & 287 & -1491 & 286 & -1823 & 296 \\
\hline-1235 & 310 & -1333 & 287 & -1492 & 305 & -1824 & 296 \\
\hline-1236 & 310 & -1334 & 287 & -1493 & 318 & -1825 & 296 \\
\hline-1237 & 310 & -1335 & 311 & -1496 & 306 & -1828 & 312 \\
\hline-1239 & 316 & -1336 & 311 & -1497 & 305 & -1829 & 312 \\
\hline-1248 & 281 & -1337 & 311 & -1498 & 305 & -1830 & 312 \\
\hline-1253 & 291 & -1338 & 311 & -1499 & 305 & -1831 & 312 \\
\hline-1256 & 311 & -1339 & 315 & -1500 & 305 & -1915 & 296 \\
\hline-1257 & 312 & -1340 & 316 & -1501 & 305 & -1916 & 296 \\
\hline-1258 & 312 & -1341 & 315 & -1502 & 304 & -1942 & 316 \\
\hline-1259 & 303 & -1342 & 316 & -1513 & 280 & & \\
\hline-1261 & 307 & -1343 & 316 & -1514 & 280 & GrN & \\
\hline-1262 & 307 & -1344 & 316 & -1529 & 306 & -611 & 42 \\
\hline-1263 & 307 & -1345 & 280 & -1549 & 314 & -677 & 103 \\
\hline-1265 & 298 & -1346 & 280 & -1550 & 314 & -680 & 103 \\
\hline-1266 & 290 & -1347 & 280 & -1551 & 315 & -823 & 42 \\
\hline-1267 & 317 & -1348 & 280 & -1552 & 305 & -836 & 104 \\
\hline-1268 & 317 & -1349 & 300 & -1553 & 305 & -850 & 29 \\
\hline-1269 & 317 & -1354 & 289 & -1570 & 313 & -854 & 44 \\
\hline-1270 & 317 & -1359 & 284 & -1571 & 313 & -1111 & 103 \\
\hline-1271 & 304 & -1360 & 285 & -1572 & 313 & -1435 & 7 \\
\hline-1272 & 300 & -1361 & 296 & -1573 & 313 & -1544 & 50 \\
\hline-1273 & 285 & -1364 & 295 & -1581 & 304 & -1583 & 40 \\
\hline-1274 & 285 & -1365 & 295 & -1605 & 316 & -1585 & 36 \\
\hline-1275 & 284 & -1367 & 294 & -1621 & 287 & -1586 & 36 \\
\hline-1278 & 306 & -1369 & 295 & -1625 & 305 & -1593 & 36 \\
\hline-1282 & 295 & -1370 & 295 & -1626 & 305 & -1594 & 36 \\
\hline-1284 & 295 & -1371 & 295 & -1631 & 288 & -1595 & 36 \\
\hline-1285 & 295 & -1375 & 306 & -1632 & 288 & -1698 & 12 \\
\hline-1286 & 295 & -1389 & 308 & -1633 & 289 & -1711 & 7 \\
\hline-1287 & 295 & -1390 & 308 & -1634 & 289 & -1713 & 7 \\
\hline-1291 & 296 & -1391 & 308 & -1635 & 289 & -1760 & 15 \\
\hline-1292 & 296 & -1408 & 304 & -1646 & 315 & -1761 & 14 \\
\hline-1293 & 302 & -1409 & 304 & -1647 & 315 & -1799 & 7 \\
\hline-1294 & 302 & -1410 & 304 & -1648 & 314 & -1807 & 7 \\
\hline-1295 & 302 & -1413 & 319 & -1649 & 314 & -1894 & 104 \\
\hline-1303 & 282 & -1434 & 289 & -1650 & 314 & -1895 & 104 \\
\hline-1304 & 281 & -1442 & 312 & -1651 & 314 & -1921 & 7 \\
\hline-1305 & 307 & -1443 & 312 & -1652 & 314 & -1922 & 11 \\
\hline-1306 & 307 & -1447 & 301 & -1663 & 301 & -1924 & 11 \\
\hline-1312 & 283 & -1448 & 301 & -1664 & 302 & -1925 & 12 \\
\hline-1313 & 282 & -1462 & 283 & -1665 & 302 & -1926 & 11 \\
\hline-1314 & 283 & -1463 & 281 & -1666 & 301 & -1949 & 30 \\
\hline-1315 & 281 & -1464 & 282 & -1691 & 314 & -1950 & 30 \\
\hline-1316 & 293 & -1466 & 281 & -1692 & 314 & -2043 & 45 \\
\hline-1318 & 293 & -1467 & 283 & -1693 & 314 & -2145 & 30 \\
\hline-1319 & 310 & -1471 & 318 & -1713 & 319 & -2146 & 30 \\
\hline-1320 & 310 & -1472 & 297 & -1746 & 313 & -2147 & 30 \\
\hline-1321 & 310 & -1473 & 297 & -1747 & 313 & -2185 & 30 \\
\hline-1322 & 310 & -1474 & 297 & -1749 & 314 & -2187 & 30 \\
\hline-1323 & 310 & -1475 & 297 & -1750 & 314 & -2191 & 30 \\
\hline-1324 & 297 & -1479 & 311 & -1751 & 315 & -2221 & 85 \\
\hline-1325 & 297 & -1484 & 286 & -1752 & 315 & -2327 & 30 \\
\hline-1326 & 297 & -1485 & 286 & -1753 & 315 & -2379 & 40 \\
\hline-1327 & 318 & -1486 & 286 & -1754 & 314 & -2382 & 41 \\
\hline-1328 & 288 & -1487 & 286 & -1755 & 314 & -2386 & 40 \\
\hline-1329 & 283 & -1488 & 286 & -1766 & 316 & -2389 & 40 \\
\hline
\end{tabular}




\begin{tabular}{|c|c|c|c|c|c|c|c|}
\hline $\begin{array}{c}\text { Sample } \\
\text { no. }\end{array}$ & $\begin{array}{c}\text { Page } \\
\text { no. }\end{array}$ & $\begin{array}{c}\text { Sample } \\
\text { no. }\end{array}$ & $\begin{array}{c}\text { Page } \\
\text { no. }\end{array}$ & $\begin{array}{c}\text { Sample } \\
\text { no. }\end{array}$ & $\begin{array}{c}\text { Page } \\
\text { no. }\end{array}$ & $\begin{array}{c}\text { Sample } \\
\text { no. }\end{array}$ & $\begin{array}{c}\text { Page } \\
\text { no. }\end{array}$ \\
\hline GrN & & GrN & & GrN & & GrN & \\
\hline-2406 & 26 & -4211 & 101 & -4514 & 14 & -4897 & 55 \\
\hline-2499 & 41 & -4212 & 101 & -4543 & 25 & -4913 & 33 \\
\hline-2555 & 8 & -4219 & 26 & -4600 & 39 & -4914 & 33 \\
\hline-2565 & 12 & -4220 & 26 & -4601 & 39 & -4915 & 33 \\
\hline-2568 & 18 & -4232 & 104 & -4602 & 19 & -4916 & 33 \\
\hline-2570 & 9 & -4233 & 80 & -4603 & 19 & -4917 & 33 \\
\hline-2580 & 9 & -4235 & 103 & -4614 & 15 & -4918 & 33 \\
\hline-2601 & 9 & -4237 & 8 & -4619 & 40 & -4922 & 81 \\
\hline-2619 & 10 & -4238 & 10 & -4620 & 41 & -4937 & 48 \\
\hline-2625 & 10 & -4263 & 32 & -4621 & 41 & -4938 & 48 \\
\hline-2630 & 30 & -4264 & 32 & -4622 & 97 & -4939 & 48 \\
\hline-2632 & 12 & -4265 & 32 & -4623 & 35 & -4943 & 31 \\
\hline-2633 & 12 & -4266 & 32 & -4624 & 35 & -4945 & 96 \\
\hline-2634 & 12 & -4267 & 32 & -4625 & 34 & -4946 & 96 \\
\hline-2657 & 30 & -4272 & 11 & -4626 & 35 & -4947 & 97 \\
\hline-2658 & 30 & -4274 & 33 & -4630 & 22 & -4948 & 85 \\
\hline-2837 & 45 & -4275 & 33 & -4635 & 88 & -4950 & 63 \\
\hline-2839 & 45 & -4276 & 32 & -4658 & 63 & -4951 & 43 \\
\hline-2850 & 45 & -4277 & 32 & -4659 & 63 & -4952 & 43 \\
\hline-2880 & 59 & -4278 & 32 & -4660 & 63 & -4954 & 44 \\
\hline-2889 & 58 & -4279 & 32 & -4661 & 63 & -4955 & 43 \\
\hline-2895 & 59 & -4299 & 59 & -4665 & 98 & -4956 & 31 \\
\hline-2913 & 59 & -4301 & 33 & -4677 & 57 & -4957 & 94 \\
\hline-2940 & 105 & -4302 & 32 & -4680 & 22 & -4958 & 94 \\
\hline-2943 & 106 & -4303 & 32 & -4693 & 57 & -4959 & 96 \\
\hline-2952 & 30 & -4312 & 34 & -4712 & 27 & -4960 & 58 \\
\hline-2955 & 30 & -4313 & 33 & -4744 & 17 & -4961 & $\begin{array}{l}50 \\
58\end{array}$ \\
\hline-2968 & 104 & -4314 & 33 & -4760 & 22 & -4966 & $\begin{array}{l}58 \\
67\end{array}$ \\
\hline-2975 & 41 & -4371 & 39 & -4793 & 29 & -4967 & 100 \\
\hline-2981 & 26 & -4383 & 57 & -4798 & 17 & -4968 & 99 \\
\hline-3026 & 104 & -4397 & 9 & -4799 & 9 & -4969 & 100 \\
\hline-3029 & 104 & -4398 & 13 & -4800 & 9 & -4971 & 16 \\
\hline-3040 & 104 & -4399 & 54 & -4801 & 34 & -4976 & 66 \\
\hline-3089 & 56 & -4400 & 54 & -4802 & 34 & -4977 & 66 \\
\hline-3163 & 12 & -4401 & 46 & -4803 & 34 & -4978 & 66 \\
\hline-3213 & 12 & -4402 & 46 & -4805 & 31 & -4979 & 60 \\
\hline-3219 & 13 & -4403 & 46 & -4807 & 27 & -4980 & 60 \\
\hline-3546 & 42 & -4404 & 49 & -4808 & 31 & -4983 & 60 \\
\hline-3549 & 56 & -4408 & 15 & -4811 & 11 & -4984 & 61 \\
\hline-4064 & 19 & -4411 & 46 & -4817 & 8 & -4987 & 43 \\
\hline-4074 & 16 & -4415 & 14 & -4833 & 98 & -4988 & 61 \\
\hline-4086 & 17 & -4423 & 54 & -4837 & 47 & -4989 & 61 \\
\hline-4094 & 16 & -4424 & 70 & -4838 & 47 & -4990 & 60 \\
\hline-4098 & 27 & -4425 & 71 & -4840 & 80 & -4995 & 25 \\
\hline-4102 & 27 & -4429 & 10 & -4841 & 47 & -4996 & 13 \\
\hline-4114 & 84 & -4434 & 18 & -4842 & 47 & -4998 & 22 \\
\hline-4116 & 16 & -4435 & 18 & -4843 & 47 & -4999 & 23 \\
\hline-4132 & 15 & -4436 & 18 & -4844 & 47 & -5000 & 64 \\
\hline-4133 & 14 & -4448 & 17 & -4845 & 28 & -5001 & 97 \\
\hline-4139 & 14 & -4454 & 8 & -4846 & 28 & -5007 & 54 \\
\hline-4146 & 35 & -4468 & 15 & -4849 & 28 & -5009 & 56 \\
\hline-4148 & 35 & -4476 & 102 & -4853 & 45 & -5011 & 23 \\
\hline-4165 & 17 & -4479 & 62 & -4867 & 24 & -5012 & 56 \\
\hline-4180 & 80 & -4491 & 25 & -4868 & 24 & -5013 & 66 \\
\hline-4181 & 81 & -4492 & 44 & -4873 & 45 & -5026 & 31 \\
\hline-4182 & 28 & -4496 & 21 & -4874 & 46 & -5027 & 86 \\
\hline-4183 & 28 & -4497 & 24 & -4875 & 29 & -5028 & 86 \\
\hline-4184 & 28 & -4512 & 14 & -4894 & 55 & -5029 & 86 \\
\hline-4205 & 81 & -4513 & 14 & -4895 & 55 & -5030 & 96 \\
\hline
\end{tabular}




\begin{tabular}{|c|c|c|c|c|c|c|c|}
\hline $\begin{array}{c}\text { Sample } \\
\text { no. }\end{array}$ & $\begin{array}{c}\text { Page } \\
\text { no. }\end{array}$ & $\begin{array}{c}\text { Sample } \\
\text { no. }\end{array}$ & $\begin{array}{c}\text { Page } \\
\text { no. }\end{array}$ & $\begin{array}{c}\text { Sample } \\
\text { no. }\end{array}$ & $\begin{array}{c}\text { Page } \\
\text { no. }\end{array}$ & $\begin{array}{c}\text { Sample } \\
\text { no. }\end{array}$ & $\begin{array}{c}\text { Page } \\
\text { no. }\end{array}$ \\
\hline GrN & & GrN & & GrN & & GrN & \\
\hline-5031 & 85 & -5165 & 37 & -5352 & 93 & -5.542 & 25 \\
\hline-5034 & 89 & $-5 \mathrm{I} 66$ & 37 & -5353 & 44 & -5543 & 25 \\
\hline-5035 & 69 & -5167 & 105 & -5.354 & 43 & -5549 & 83 \\
\hline-5036 & 70 & -5168 & 105 & -5355 & 43 & -5552 & 42 \\
\hline-5037 & 75 & -5169 & 10.5 & -5356 & 94 & -5553 & 41 \\
\hline-5038 & 80 & -5175 & 85 & -5.357 & 94 & -5554 & 41 \\
\hline-5043 & 81 & -5176 & 87 & -5375 & 19 & -5555 & 41 \\
\hline-5044 & 69 & -5177 & 88 & -5376 & 92 & -5566 & 87 \\
\hline-5045 & 69 & -5180 & 36 & -5377 & 38 & -5567 & 25 \\
\hline-5046 & 70 & -5181 & 64 & -5378 & 38 & -5568 & 78 \\
\hline-5047 & 75 & -5183 & 90 & -5379 & 38 & -5573 & 93 \\
\hline-5048 & 96 & -5184 & 90 & -5380 & 38 & -5574 & 92 \\
\hline-5050 & 95 & -5185 & 91 & -5382 & 100 & -5576 & 49 \\
\hline-5051 & 95 & -5186 & 90 & -5384 & 68 & -5577 & 52 \\
\hline-5062 & 50 & -5187 & 90 & -5390 & 83 & -5578 & 52 \\
\hline-5063 & 50 & -5191 & 21 & -5391 & 83 & -5580 & 51 \\
\hline-5067 & 82 & -5195 & 98 & -5393 & 57 & -5581 & 51 \\
\hline-5068 & 86 & -5196 & 98 & -5404 & 19 & -5588 & 91 \\
\hline-5069 & 86 & -5197 & 52 & -5405 & 19 & -5.590 & 101 \\
\hline-5070 & 84 & -5198 & 52 & -5408 & 21 & -5.591 & 99 \\
\hline-5071 & 23 & -5199 & 52 & -5409 & 67 & -5592 & 99 \\
\hline-5077 & 48 & -5200 & 52 & -5410 & 67 & -5593 & 74 \\
\hline-5081 & 82 & -5201 & 52 & -5419 & 76 & -5594 & 75 \\
\hline-.5082 & 82 & -5202 & 44 & -5420 & 76 & -5595 & 75 \\
\hline-5085 & 23 & -.5203 & 43 & -5421 & 77 & -5596 & 75 \\
\hline-5086 & 79 & -5215 & 55 & -5422 & 76 & -5597 & 74 \\
\hline-5087 & 69 & -5216 & 21 & -5425 & 65 & -5598 & 75 \\
\hline-5088 & 69 & -5217 & 95 & -5442 & 68 & -5601 & 72 \\
\hline-5089 & 70 & -5218 & 93 & -5443 & 82 & -5602 & 74 \\
\hline-5090 & 70 & -5219 & 93 & -5445 & 79 & -5603 & 76 \\
\hline-5097 & 66 & -5220 & 93 & -5446 & 65 & -5604 & 72 \\
\hline-5098 & 65 & -5221 & 93 & -5447 & 48 & -5606 & 82 \\
\hline-.5100 & 65 & -5238 & 39 & -5448 & 48 & -5612 & 79 \\
\hline-5102 & 55 & -.5241 & 53 & -5449 & 48 & -5619 & 37 \\
\hline-5103 & 84 & -5249 & 80 & -5450 & 49 & -5620 & 37 \\
\hline-5105 & 91 & -5250 & 77 & -.5451 & 48 & -5621 & 37 \\
\hline-5108 & 78 & -5251 & 68 & -.5459 & 38 & -5626 & 102 \\
\hline-5109 & 78 & -5253 & 67 & -5460 & 20 & -5627 & 102 \\
\hline-5110 & 73 & -5254 & 68 & -5461 & 101 & -5628 & 73 \\
\hline-5111 & 24 & -5255 & 67 & -5462 & 73 & -5629 & 79 \\
\hline-5125 & 72 & -5257 & 16 & -5463 & 74 & -5633 & 53 \\
\hline-5126 & 71 & -5264 & 88 & -5480 & 20 & -5634 & 53 \\
\hline-5129 & 20 & -.5265 & 91 & -5481 & 95 & -5646 & 100 \\
\hline-5130 & 62 & -.5266 & 94 & -5482 & 95 & -5705 & 87 \\
\hline-5131 & 87 & -5281 & 22 & -5485 & 98 & -5706 & 58 \\
\hline-5132 & 87 & -5283 & 53 & -5486 & 13 & -5734 & 72 \\
\hline-5133 & 78 & -5284 & 51 & -5489 & 101 & -5764 & 74 \\
\hline-5134 & 71 & -5285 & 51 & -5490 & 24 & -5767 & 84 \\
\hline-5135 & 77 & -5286 & 51 & -5491 & 99 & -5775 & 92 \\
\hline-5136 & 50 & -5287 & 51 & -.5494 & 89 & -5776 & 92 \\
\hline-5139 & 84 & -5288 & 65 & -5495 & 89 & -5777 & 91 \\
\hline-5141 & 20 & -5295 & 87 & -5496 & 88 & -5861 & 91 \\
\hline-5142 & 20 & -5327 & 38 & -5497 & 89 & $\left.-586^{\circ}\right)$ & 91 \\
\hline-.5143 & 38 & -5328 & 38 & -5498 & 89 & $\begin{array}{r}-1.9802 \\
5802\end{array}$ & 91 \\
\hline-5156 & 77 & -5329 & 39 & -5499 & 57 & -5895 & 89 \\
\hline-5157 & 102 & -5348 & 83 & -5505 & 98 & -6058 & 62 \\
\hline-5158 & 103 & -5349 & 83 & -5512 & 58 & -6059 & 64 \\
\hline-5163 & 37 & -5350 & 89 & -5513 & 59 & -6083 & 61 \\
\hline-5164 & 37 & -5351 & 92 & .5514 & 59 & -6097 & 104 \\
\hline
\end{tabular}




\begin{tabular}{|c|c|c|c|c|c|c|c|}
\hline $\begin{array}{c}\text { Sample } \\
\text { no. }\end{array}$ & $\begin{array}{c}\text { Page } \\
\text { no. }\end{array}$ & $\begin{array}{c}\text { Sample } \\
\text { no. }\end{array}$ & $\begin{array}{c}\text { Page } \\
\text { no. }\end{array}$ & $\begin{array}{c}\text { Sample } \\
\text { no. }\end{array}$ & $\begin{array}{c}\text { Page } \\
\text { no. }\end{array}$ & $\begin{array}{c}\text { Sample } \\
\text { no. }\end{array}$ & $\begin{array}{c}\text { Page } \\
\text { no. }\end{array}$ \\
\hline $\mathrm{Gu}$ & & $\mathrm{Gu}$ & & $\mathrm{Gu}$ & & I & \\
\hline-300 & 111 & -359 & 323 & -423 & 328 & -4212 & 116 \\
\hline-301 & 111 & -360 & 323 & -424 & 329 & -4213 & 116 \\
\hline-302 & 111 & -361 & 323 & -425 & 329 & -4214 & 116 \\
\hline-303 & 111 & -362 & 323 & -426 & 329 & -4215 & 116 \\
\hline-304 & 111 & -363 & 323 & -427 & 329 & -4234 & 132 \\
\hline-305 & 112 & -364 & 323 & -428 & 329 & -4267 & 125 \\
\hline-306 & 112 & -365 & 323 & -429 & 329 & -4286 & 135 \\
\hline-307 & 112 & -266 & 324 & -430 & 329 & -4287 & 135 \\
\hline-308 & 112 & -367 & 324 & -431 & 329 & -4306 & 134 \\
\hline-309 & 112 & -368 & 324 & -432 & 329 & -4310 & 133 \\
\hline-310 & 112 & -369 & 324 & -433 & 329 & -4311 & 133 \\
\hline-311 & 112 & -370 & 324 & -434 & 329 & -4364 & 125 \\
\hline-312 & 112 & -371 & 324 & -435 & 329 & -4366 & 126 \\
\hline-313 & 112 & -372 & 324 & -436 & 329 & -4367 & 126 \\
\hline-314 & 112 & -373 & 324 & -437 & 329 & -4368 & 137 \\
\hline-315 & 112 & -374 & 324 & -438 & 329 & -4369 & 137 \\
\hline-316 & 112 & -375 & 324 & -439 & 329 & -4370 & 137 \\
\hline-317 & 112 & -381 & 327 & -440 & 329 & -4375 & 129 \\
\hline-318 & 112 & -382 & 327 & -441 & 330 & -4376 & 129 \\
\hline-319 & 112 & -383 & 327 & -442 & 330 & -4378 & 126 \\
\hline-320 & 112 & -384 & 327 & -443 & 330 & -4404 & 135 \\
\hline-321 & 112 & -885 & 327 & -444 & 330 & -4405 & 135 \\
\hline-322 & 112 & -386 & 327 & -445 & 330 & -4406 & 135 \\
\hline-323 & 112 & -387 & 327 & -446 & 330 & -4409 & 115 \\
\hline-324 & 113 & -388 & 327 & -447 & 330 & -4444 & 126 \\
\hline-325 & 113 & -389 & 327 & -448 & 330 & -4445 & 126 \\
\hline-326 & 113 & -390 & 327 & -449 & 330 & -4446 & 126 \\
\hline-327 & 113 & -391 & 327 & -450 & 330 & -4447 & 131 \\
\hline-328 & 113 & -392 & 327 & -451 & 330 & -4449 & 136 \\
\hline $\begin{array}{r}-329 \\
\end{array}$ & 113 & -393 & 327 & & & -4455 & 125 \\
\hline-330 & 113 & -394 & 327 & I & & -4513 & 132 \\
\hline-331 & 113 & -395 & 327 & -2982 & 116 & -4514 & 132 \\
\hline-332 & 113 & -396 & 327 & -3056 & 114 & -4515 & 125 \\
\hline-333 & 113 & -397 & 327 & -3057 & 114 & -4528 & 123 \\
\hline-334 & 321 & -398 & 327 & -3654 & 115 & -4542 & 132 \\
\hline-335 & 321 & -399 & 327 & -3656 & 115 & -4550 & 129 \\
\hline-336 & 321 & -400 & 327 & -3677 & 114 & -4557 & 126 \\
\hline-337 & 321 & -401 & 327 & -3770 & 117 & -4558 & 125 \\
\hline-338 & 322 & -402 & 327 & -3771 & 117 & -4568 & 123 \\
\hline-339 & 322 & -403 & 327 & -3772 & 117 & -4569 & 124 \\
\hline-340 & 322 & -404 & 327 & -3773 & 117 & -4570 & 124 \\
\hline-341 & 322 & -405 & 327 & -3911 & 131 & -4571 & 124 \\
\hline-342 & 322 & -406 & 327 & -3962 & 123 & -4572 & 124 \\
\hline-343 & 322 & -407 & 327 & -3963 & 123 & -4573 & 124 \\
\hline-344 & 322 & -408 & 327 & -3971 & 117 & -4647 & 116 \\
\hline-345 & 322 & -409 & 327 & -3972 & 117 & -4648 & 116 \\
\hline-346 & 322 & -410 & 327 & -3977 & 121 & -4684 & 130 \\
\hline-347 & 322 & -411 & 327 & -4101 & 121 & -4685 & 130 \\
\hline-348 & 322 & -412 & 328 & -4102 & 121 & -4686 & 130 \\
\hline-349 & 322 & -413 & 328 & -4103 & 121 & -4687 & 130 \\
\hline-350 & 323 & -414 & 328 & -4104 & 122 & -4688 & 130 \\
\hline-351 & 323 & -415 & 328 & -4120 & 133 & -4718 & 134 \\
\hline-352 & 323 & -416 & 328 & -4121 & 133 & -4730 & 120 \\
\hline-353 & 323 & -417 & 328 & -4122 & 133 & -4756 & 122 \\
\hline-354 & 323 & -418 & 328 & -4137 & 127 & -4782 & 122 \\
\hline-355 & 323 & -419 & 328 & -4163 & 115 & -4798 & 123 \\
\hline-356 & 323 & -420 & 328 & -4199 & 133 & -4801 & 120 \\
\hline-357 & 323 & -421 & 328 & -4200 & 133 & -4802 & 120 \\
\hline-358 & 323 & -422 & 328 & -4201 & 133 & -4823 & 134 \\
\hline
\end{tabular}




\begin{tabular}{|c|c|c|c|c|c|c|c|}
\hline $\begin{array}{c}\text { Sample } \\
\text { no. }\end{array}$ & $\begin{array}{c}\text { Page } \\
\text { no. }\end{array}$ & $\begin{array}{c}\text { Sample } \\
\text { no. }\end{array}$ & $\begin{array}{c}\text { Page } \\
\text { no. }\end{array}$ & $\begin{array}{c}\text { Sample } \\
\text { no. }\end{array}$ & $\begin{array}{c}\text { Page } \\
\text { no. }\end{array}$ & $\begin{array}{c}\text { Sample } \\
\text { no. }\end{array}$ & $\begin{array}{c}\text { Page } \\
\text { no. }\end{array}$ \\
\hline I & & IGS & & IRPA & & LE & \\
\hline-4824 & 134 & $-\mathrm{C} 14 / 26$ & 141 & -85 & 146 & -753 & 337 \\
\hline-4825 & 134 & $-\mathrm{Cl} 14 / 27$ & 142 & -86 & 146 & -754 & 337 \\
\hline-4826 & 134 & $-\mathrm{C} 14 / 28$ & 142 & -87 & 146 & -755 & 337 \\
\hline-4827 & 134 & $-\mathrm{C} 14 / 29$ & 142 & -88 & 147 & -756 & 337 \\
\hline-4835 & 126 & $-\mathrm{C} 14 / 30$ & 142 & -89 & 147 & -757 & 337 \\
\hline-4836 & 127 & $-\mathrm{C} 14 / 31$ & 142 & & & -758 & 338 \\
\hline-4837 & 127 & $-\mathrm{C} 14 / 32$ & 143 & ISGS & & -760 & 359 \\
\hline-4838 & 127 & $-\mathrm{C} 14 / 33$ & 143 & -33 & 149 & -761 & 359 \\
\hline-4862 & 131 & $-\mathrm{Cl} 14 / 34$ & $\mathrm{I} 43$ & -34 & 153 & -762 & 359 \\
\hline-4869 & 131 & $-\mathrm{Cl} 14 / 35$ & 143 & -35 & 154 & -763 & 359 \\
\hline-4879 & 128 & $-\mathrm{C} 14 / 36$ & 143 & -36 & 149 & -764 & 359 \\
\hline-4880 & 128 & $-\mathrm{C} 14 / 37$ & 143 & -37 & 150 & -766 & 348 \\
\hline-4881 & 128 & $-\mathrm{C} 14 / 38$ & 143 & -38 & 151 & -767 & 351 \\
\hline-4899 & 118 & $-\mathrm{C} 14 / 39$ & 143 & -39 & 150 & -768 & 349 \\
\hline-4900 & 118 & $-\mathrm{CI} 4 / 40$ & 144 & -40 & 152 & -769 & 351 \\
\hline-4910 & 115 & $-\mathrm{C} 14 / 41$ & 144 & -41 & 152 & -770 & 351 \\
\hline-4926 & 135 & -CI4/42 & 331 & -42 & 152 & -771 & 356 \\
\hline-4961 & 134 & $-\mathrm{C} 14 / 46$ & 331 & -43 & 153 & -772 & 354 \\
\hline-4963 & 120 & -CI4/47 & 331 & -44 & 153 & -773 & 354 \\
\hline-4972 & 130 & $-\mathrm{Cl} 4 / 48$ & 331 & -45 & 150 & -775 & 360 \\
\hline$-4985 \mathrm{~A}$ & 131 & $-\mathrm{C} 14 / 49$ & 331 & -46 & 150 & -776 & 348 \\
\hline$-4985 \mathrm{~B}$ & 131 & $-\mathrm{C} 14 / 52$ & 332 & -47 & 150 & -777 & 354 \\
\hline-5070 & 120 & $-\mathrm{C} 14 / 53$ & 332 & -48 & 154 & -779 & 351 \\
\hline-5078 & 118 & -C14/54 & 332 & -49 & 151 & -780 & 350 \\
\hline-5079 & 118 & $-\mathrm{C} 14 / 55$ & 332 & -50 & 151 & -781 & 351 \\
\hline-5080 & 119 & $-\mathrm{C} 14 / 56$ & 332 & -51 & 154 & -784 & 357 \\
\hline-5081 & 119 & $-\mathrm{Cl} 14 / 57$ & 333 & -52 & 151 & -787 & 354 \\
\hline-5082 & 119 & $-\mathrm{C} 14 / 58$ & 333 & -53 & 150 & -788 & 355 \\
\hline-5083 & 122 & $-\mathrm{C} 14 / 59$ & 333 & -54 & 153 & -791 & 356 \\
\hline-5088 & 124 & $-\mathrm{C} 14 / 60$ & 334 & -55 & 152 & -792 & 356 \\
\hline-5089 & 124 & $-\mathrm{C} 14 / 61$ & 334 & -56 & 152 & -793 & 357 \\
\hline-5090 & 125 & $-\mathrm{C} 14 / 62$ & 334 & -57 & 152 & -799 & 336 \\
\hline-5091 & 125 & $-\mathrm{Cl} 4 / 64$ & 334 & -59 & 159 & -800 & 341 \\
\hline-5177 & 120 & $-\mathrm{C} 14 / 65$ & 334 & & & -801 & 359 \\
\hline-5178 & 121 & $-\mathrm{C} 14 / 66$ & 334 & LE & & -802 & 359 \\
\hline-5179 & 121 & $-\mathrm{C} 14 / 67$ & 334 & -355 & 350 & -808 & 358 \\
\hline-5236 & 127 & $-\mathrm{C} 14 / 68$ & 141 & -720 & 355 & -809 & 359 \\
\hline-5249 & 128 & & & -721 & 355 & -810 & 338 \\
\hline-5250 & 128 & IRPA & & -722 & 355 & -811 & 338 \\
\hline-5251 & 128 & -49 & 147 & -723 & 355 & -812 & 398 \\
\hline-5252 & 129 & -51 & 147 & -724 & 356 & -813 & 340 \\
\hline-.5253 & 129 & -52 & 147 & -725 & 348 & -814 & 340 \\
\hline-5254 & 129 & -66 & 145 & -726 & 349 & -815 & 340 \\
\hline-5318 & 137 & -67 & 145 & -727 & 349 & -816 & 340 \\
\hline-5319 & 136 & -68 & 145 & -730 & 362 & -818 & 351 \\
\hline-5320 & 136 & -69 & 145 & -731 & 350 & -822 & 349 \\
\hline-5321 & 136 & -70 & 147 & -740 & 359 & -823 & 349 \\
\hline-5475 & 136 & -71 & 147 & -741 & 360 & -824 & 349 \\
\hline-5476 & 136 & -72 & 147 & -742 & 360 & -825 & 362 \\
\hline-5477 & 137 & -73 & 148 & -743 & 359 & -826 & 362 \\
\hline & & -74 & 148 & -744 & 359 & -829 & 357 \\
\hline IGS & & -75 & 148 & -745 & 359 & -830 & 362 \\
\hline$-\mathrm{C} 14 / 16$ & 140 & -76 & 146 & -746 & 359 & -831 & 361 \\
\hline$-\mathrm{C} 14 / 17$ & 140 & -77 & 146 & -747 & 359 & -832 & 361 \\
\hline$-\mathrm{C} 14 / 18$ & 140 & -78 & 146 & -748 & 338 & -833 & 341 \\
\hline$-\mathrm{C} 14 / 19$ & 140 & -79 & 146 & -749 & 339 & -834 & 339 \\
\hline$-\mathrm{C} 14 / 20$ & 141 & -80 & 146 & -750 & 338 & -835 & 341 \\
\hline$-\mathrm{C} 14 / 21$ & 141 & -81 & 146 & -751 & 339 & -836 & 339 \\
\hline$. \mathrm{CI} 4 / 25$ & 142 & -84 & 146 & -752 & 339 & -837 & 339 \\
\hline
\end{tabular}




\begin{tabular}{|c|c|c|c|c|c|c|c|}
\hline $\begin{array}{c}\text { Sample } \\
\text { no. }\end{array}$ & $\begin{array}{c}\text { Page } \\
\text { no. }\end{array}$ & $\begin{array}{c}\text { Sample } \\
\text { no. }\end{array}$ & $\begin{array}{c}\text { Page } \\
\text { no. }\end{array}$ & $\begin{array}{c}\text { Sample } \\
\text { no. }\end{array}$ & $\begin{array}{c}\text { Page } \\
\text { no. }\end{array}$ & $\begin{array}{c}\text { Sample } \\
\text { no. }\end{array}$ & $\begin{array}{c}\text { Page } \\
\text { no. }\end{array}$ \\
\hline LE & & LJ & & $\mathbf{L u}$ & & $\mathrm{Lu}$ & \\
\hline-838 & 339 & -2190 & 377 & -487 & 388 & -596 & 383 \\
\hline-850 & 338 & -2191 & 377 & -488 & 388 & -597 & 383 \\
\hline \multirow[t]{2}{*}{-904} & 341 & -2195 & 377 & -489 & 388 & & \\
\hline & & -2322 & 377 & -490 & 389 & M & \\
\hline LJ & & -2323 & 377 & -491 & 389 & -1190 & 204 \\
\hline -GAP0093 & $3 \quad 374$ & -2324 & 377 & -492 & 389 & -1191 & 205 \\
\hline-1625 & 374 & -2342 & 373 & -493 & 383 & -1192 & 205 \\
\hline-1627 & 374 & & & -494 & 384 & -1193 & 205 \\
\hline-1628 & 375 & Lu & & -495 & 384 & -1194 & 205 \\
\hline-1629 & 375 & -400 & 381 & -496 & 384 & -1195 & 205 \\
\hline-1630 & 375 & -401 & 381 & -497 & 384 & -1325 & 188 \\
\hline-1631 & 375 & -402 & 381 & -498 & 384 & -1420 & 215 \\
\hline-1632 & 375 & -403 & 381 & -499 & 384 & -1639 & 218 \\
\hline-1633 & 375 & -404 & 381 & -500 & 384 & -1725 & 200 \\
\hline-1634 & 375 & -405 & 381 & -501 & 384 & -1726 & 201 \\
\hline-1635 & 376 & -425 & 385 & -502 & 398 & -1728 & 201 \\
\hline-1636 & 376 & -436 & 394 & -504 & 384 & -1729 & 201 \\
\hline-1637 & 376 & -437 & 396 & -505 & 387 & -1730 & 201 \\
\hline-1904 & 376 & -438 & 390 & $-505 A$ & 387 & -1764 & 216 \\
\hline-1905 & 376 & -439 & 390 & -506 & 387 & -1765 & 216 \\
\hline-1906 & 374 & -440 & 391 & $-506 \mathrm{~A}$ & 388 & -1771 & 216 \\
\hline-1907 & 374 & -441 & 391 & -507 & 386 & -1773 & 216 \\
\hline-1982 & 373 & -442 & 391 & -516 & 399 & -1774 & 217 \\
\hline-1984 & 373 & -443 & 391 & -517 & 399 & -1784 & 208 \\
\hline-1990 & 373 & -444 & 391 & -518 & 399 & -1785 & 209 \\
\hline-1992 & 373 & -446 & 385 & -519 & 399 & -1844 & 191 \\
\hline-1994 & 373 & -451 & 397 & -520 & 399 & $-1844 a$ & 191 \\
\hline-2032 & 369 & -452 & 398 & -521 & 399 & -1870 & 202 \\
\hline-2037 & 369 & -453 & 396 & -524 & 395 & -1871 & 202 \\
\hline-2040 & 369 & -454 & 396 & -525 & 395 & -1872 & 202 \\
\hline-2042 & 370 & -455 & 396 & -526 & 380 & -1873 & 202 \\
\hline-2044 & 370 & -457 & 392 & -527 & 381 & -1875 & 202 \\
\hline-2047 & 370 & -458 & 392 & -528 & 389 & -1876 & 202 \\
\hline-2048 & 370 & -459 & 393 & -529 & 389 & -1877 & 202 \\
\hline-2053 & 371 & -460 & 393 & -530 & 389 & -1879 & 202 \\
\hline-2059 & 371 & -461 & 393 & -531 & 390 & -1881 & 202 \\
\hline-2061 & 371 & -462 & 393 & -540 & 383 & -1882 & 202 \\
\hline-2068 & 371 & -463 & 393 & -541 & 383 & -1883 & 202 \\
\hline-2070 & 371 & -464 & 393 & -542 & 383 & -1884 & 203 \\
\hline-2075 & 371 & -465 & 393 & -543 & 383 & -1927 & 195 \\
\hline-2078 & 371 & -469 & 385 & -544 & 397 & -1968 & 172 \\
\hline-2082 & 372 & -470 & 385 & -545 & 397 & -1970 & 200 \\
\hline-2084 & 372 & -471 & 394 & -546 & 395 & -1971 & 200 \\
\hline-2087 & 372 & -472 & 394 & -547 & 395 & -1973 & 216 \\
\hline-2090 & 372 & -473 & 394 & -548 & 395 & -1974 & 216 \\
\hline-2111 & 378 & -474 & 397 & -549 & 395 & -1975 & 196 \\
\hline-2113 & 378 & -475 & 398 & -550 & 395 & -1981 & 196 \\
\hline-2115 & 378 & -476 & 398 & -551 & 386 & -1993 & 217 \\
\hline-2116 & 378 & -477 & 398 & -562 & 392 & -2010 & 203 \\
\hline-2118 & 378 & -478 & 398 & -563 & 392 & -2015 & 157 \\
\hline-2127 & 378 & -479 & 398 & -564 & 382 & -2016 & 157 \\
\hline-2128 & 378 & -481 & 385 & -565 & 382 & -2017 & 157 \\
\hline-2130 & 378 & -482 & 390 & $-565 \mathrm{~A}$ & 382 & -2018 & 157 \\
\hline-2156 & 372 & -483 & 387 & -566 & 382 & -2019 & 157 \\
\hline-2157 & 372 & $-483 \mathrm{~A}$ & 387 & -567 & 382 & -2020 & 157 \\
\hline-2168 & 377 & -484 & 387 & -568 & 382 & -2021 & 157 \\
\hline-2181 & 373 & $-484 \mathrm{~A}$ & 387 & -577 & 392 & -2024 & 158 \\
\hline-2186 & 377 & -485 & 388 & -587 & 397 & -2028 & 218 \\
\hline-2189 & 377 & -486 & 388 & -595 & 386 & -2029 & 218 \\
\hline
\end{tabular}


Index

\begin{tabular}{|c|c|c|c|c|c|c|c|}
\hline $\begin{array}{c}\text { Sample } \\
\text { no. }\end{array}$ & $\begin{array}{c}\text { Page } \\
\text { no. }\end{array}$ & $\begin{array}{c}\text { Sample } \\
\text { no. }\end{array}$ & $\begin{array}{c}\text { Page } \\
\text { no. }\end{array}$ & $\begin{array}{c}\text { Sample } \\
\text { no. }\end{array}$ & $\begin{array}{c}\text { Page } \\
\text { no. }\end{array}$ & $\begin{array}{c}\text { Sample } \\
\text { no. }\end{array}$ & $\begin{array}{c}\text { Page } \\
\text { no. }\end{array}$ \\
\hline $\mathbf{M}$ & & M & & M & & $\mathbf{M}$ & \\
\hline-2030 & 218 & -2140 & 211 & -2213 & 173 & -2292 & 214 \\
\hline-2031 & 218 & -2141 & 211 & -2214 & 173 & -2293 & 214 \\
\hline-2032 & 219 & -2142 & 204 & -2215 & 173 & -2294 & 214 \\
\hline-2033 & 219 & -2143 & 204 & -2216 & 173 & -2295 & 214 \\
\hline-2034 & 219 & -2144 & 211 & -2217 & 173 & -2297 & 197 \\
\hline-2035 & 219 & -2145 & 156 & -2218 & 206 & -2298 & 197 \\
\hline-2042 & 162 & -2147 & 155 & -2219 & 199 & -2299 & 197 \\
\hline-2043 & 162 & -2149 & 210 & -2220 & 199 & -2300 & 197 \\
\hline-2044 & 162 & -2151 & 191 & -2221 & 199 & -2301 & 197 \\
\hline-2045 & 206 & -2152 & 191 & -2223 & 207 & -2302 & 197 \\
\hline-2050 & 198 & -2153 & 191 & -2225 & 207 & -2307 & 208 \\
\hline-2051 & 198 & -2154 & 212 & -2226 & 207 & -2308 & 208 \\
\hline-2054 & 198 & -2155 & 192 & -2229 & 207 & -2309 & 208 \\
\hline-2057 & 168 & -2156 & 192 & -2230 & 164 & -2310 & 163 \\
\hline-2058 & 168 & -2163 & 175 & -2231 & 164 & -2311 & 163 \\
\hline-2059 & 165 & -2164 & 175 & -2232 & 164 & -2312 & 163 \\
\hline-2060 & 165 & -2165 & 189 & -2233 & 164 & -2313 & 163 \\
\hline-2061 & 165 & -2166 & 189 & -2234 & 164 & -2314 & 189 \\
\hline-2062 & 165 & -2167 & 189 & -2235 & 165 & -2320 & 189 \\
\hline-2063 & 166 & -2168 & 189 & -2236 & 164 & -2321 & 190 \\
\hline-2064 & 167 & -2169 & 189 & -2237 & 165 & -2322 & 190 \\
\hline-2065 & 166 & -2170 & 193 & -2238 & 159 & -2323 & 190 \\
\hline-2075 & 210 & -2171 & 193 & -2239 & 160 & -2324 & 190 \\
\hline-2076 & 203 & -2172 & 193 & -2240 & 160 & -2325 & 190 \\
\hline-2077 & 203 & -2173 & 193 & -2241 & 160 & -2327 & 190 \\
\hline-2079 & 195 & -2175 & 175 & -2242 & 161 & -2328 & 190 \\
\hline-2080 & 216 & -2177 & 176 & -2243 & 207 & -2330 & 185 \\
\hline-2087 & 203 & -2178 & 176 & -2244 & 207 & -2331 & 185 \\
\hline-2087 & 163 & -2179 & 158 & -2245 & 208 & -2332 & 159 \\
\hline-2088 & 172 & -2180 & 177 & -2247 & 171 & -2333 & 159 \\
\hline-2102 & 186 & -2181 & 177 & -2248 & 171 & -2334 & 184 \\
\hline-2104 & 185 & -2182 & 178 & -2249 & 183 & -2335 & 197 \\
\hline-2110 & 204 & -2183 & 178 & -2251 & 183 & -2337 & 198 \\
\hline-2111 & 204 & -2184 & 178 & -2252 & 183 & -2338 & 184 \\
\hline-2112 & 172 & -2185 & 178 & -2253 & 158 & -2339 & 184 \\
\hline-2113 & 172 & -2186 & 155 & -2254 & 158 & -2340 & 184 \\
\hline-2114 & 188 & -2187 & 211 & -2255 & 171 & -2341 & 181 \\
\hline-2115 & 188 & -2188 & 212 & -2256 & 213 & $-2341 \mathrm{a}$ & 184 \\
\hline-2116 & 188 & -2189 & 212 & -2257 & 174 & $-2341 b$ & 184 \\
\hline-2117 & 188,187 & -2190 & 213 & -2258 & 174 & -2342 & 208 \\
\hline-2119 & 187 & -2191 & 213 & -2259 & 174 & -2343 & 181 \\
\hline-2120 & 187 & -2192 & 169 & -2263 & 220 & -2344 & 181 \\
\hline-2121 & 187 & -2193 & 169 & -2264 & 220 & -2345 & 181 \\
\hline-2122 & 206 & -2194 & 169 & -2265 & 220 & -2346 & 181 \\
\hline-2123 & 206 & -2195 & 169 & -2268 & 162 & -2347 & 181 \\
\hline-2124 & 214 & -2196 & 169 & -2272 & 192 & -2348 & 182 \\
\hline-2125 & 214 & -2197 & 169 & -2273 & 192 & -2349 & 182 \\
\hline-2126 & 214 & -2198 & 170 & -2274 & 178 & -2350 & 182 \\
\hline-2127 & 215 & -2200 & 170 & -2275 & 178 & -2351 & 186 \\
\hline-2128 & 215 & -2201 & 170 & -2276 & 179 & -2352 & 186 \\
\hline-2129 & 196 & -2202 & 170 & -2277 & 179 & -2353 & 186 \\
\hline-2130 & 196 & -2203 & 170 & -2278 & 179 & -2354 & 185 \\
\hline-2131 & 158 & -2204 & 170 & -2279 & 179 & -2355 & +185 \\
\hline-2133 & 177 & -2205 & 171 & -2280 & 179 & -2357 & 186 \\
\hline-2134 & 177 & -2206 & 171 & -2281 & 159 & -2358 & 186 \\
\hline-2135 & 177 & -2207 & 171 & -2286 & 193 & -2360 & 186 \\
\hline-2136 & 177 & -2208 & 156 & -2288 & 213 & -2361 & 156 \\
\hline-2137 & 177 & -2211 & 156 & -2289 & 213 & -2362 & 180 \\
\hline-2139 & 211 & -2212 & 173 & -2291 & 214 & -2363 & 180 \\
\hline
\end{tabular}




\begin{tabular}{|c|c|c|c|c|c|c|c|}
\hline $\begin{array}{c}\text { Sample } \\
\text { no. }\end{array}$ & $\begin{array}{c}\text { Page } \\
\text { no. }\end{array}$ & $\begin{array}{c}\text { Sample } \\
\text { no. }\end{array}$ & $\begin{array}{c}\text { Page } \\
\text { no. }\end{array}$ & $\begin{array}{c}\text { Sample } \\
\text { no. }\end{array}$ & $\begin{array}{c}\text { Page } \\
\text { no. }\end{array}$ & $\begin{array}{c}\text { Sample } \\
\text { no. }\end{array}$ & $\begin{array}{c}\text { Page } \\
\text { no. }\end{array}$ \\
\hline $\mathbf{M}$ & & $\mathbf{M}$ & & $\mathrm{N}$ & & $\mathrm{N}$ & \\
\hline-2364 & 180 & -2442 & 217 & -777 & 223 & -903 & 232 \\
\hline-2365 & 180 & -2455 & 190 & -778 & 224 & -904 & 232 \\
\hline-2366 & 180 & -2456 & 192 & -779 & 224 & -905 & 232 \\
\hline-2367 & 180 & -2460 & 161 & -780 & 224 & -906 & 232 \\
\hline-2368 & 181 & -2461 & 161 & -781 & 226 & -907 & 232 \\
\hline-2369 & 181 & -2462 & 162 & -784 & 236 & -908 & 232 \\
\hline-2371 & 186 & -2463 & 162 & -787 & 236 & -910 & 229 \\
\hline-2372 & 185 & -2464 & 162 & -788 & 230 & & \\
\hline-2373 & 182 & -2489 & 168 & -789 & 230 & $Q$ & \\
\hline-2374 & 182 & -2490 & 168 & -790 & 224 & -928 & 244 \\
\hline-2376 & 219 & & & -791 & 236 & -929 & 243 \\
\hline-2377 & 219 & $\mathrm{~N}$ & & -792 & 236 & -930 & 240 \\
\hline-2382 & 174 & -496 & 236 & -793 & 231 & -931 & 240 \\
\hline-2383 & 175 & -596 & 232 & -794 & 231 & -932 & 240 \\
\hline-2384 & 175 & -597 & 232 & -795 & 231 & -933 & 240 \\
\hline-2385 & 175 & -680 & 229 & -796 & 231 & -934 & 240 \\
\hline-2386 & 187 & -681 & 229 & -798 & 227 & -935 & 240 \\
\hline-2387 & 187 & -682 & 229 & -804 & 224 & -936 & 240 \\
\hline-2389 & 161 & -686 & 225 & -805 & 224 & -937 & 241 \\
\hline-2390 & 161 & -687 & 225 & -806 & 224 & -938 & 241 \\
\hline-2391 & 161 & -688 & 225 & -807 & 224 & -939 & 241 \\
\hline-2393 & 176 & -690 & 225 & -810 & 227 & -940 & 241 \\
\hline-2394 & 176 & -691 & 225 & -812 & 237 & -941 & 241 \\
\hline-2395 & 176 & -692 & 225 & -813 & 237 & -942 & 241 \\
\hline-2396 & 185 & -693 & 225 & -814 & 237 & -943 & 241 \\
\hline-2398 & 166 & -694 & 225 & -815 & 238 & -944 & 241 \\
\hline-2399 & 168 & -695 & 225 & -816 & 230 & -945 & 241 \\
\hline-2400 & 167 & -700 & 236 & -817 & 230 & -946 & 241 \\
\hline 2401 & 166 & -701 & 236 & -818 & 230 & -947 & 241 \\
\hline-2402 & 167 & -702 & 236 & -820 & 227 & -951 & 245 \\
\hline-2403 & 167 & -703 & 236 & -821 & 227 & -952 & 245 \\
\hline-2404 & 167 & -730 & 226 & $-822-1$ & 228 & -954 & 245 \\
\hline-2405 & 166 & -731 & 233 & $-822-2$ & 228 & -955 & 242 \\
\hline-2406 & 166 & -732 & 234 & $-822-3$ & 228 & -956 & 242 \\
\hline-2409 & 215 & -733 & 234 & -825 & 232 & -957 & 242 \\
\hline-2410 & 215 & -734 & 234 & -826 & 232 & -958 & 242 \\
\hline-2411 & 215 & -735 & 234 & -827 & 232 & -959 & 242 \\
\hline-2419 & 191 & -736 & 234 & -828 & 232 & -960 & 243 \\
\hline-2420 & 220 & -737 & 234 & -831 & 226 & -961 & 243 \\
\hline-2421 & 209 & -738 & 234 & -832 & 226 & -981 & 244 \\
\hline-2422 & 209 & -739 & 234 & -833 & 226 & -987 & 244 \\
\hline-2424 & 209 & -740 & 234 & -834 & 226 & -988 & 244 \\
\hline-2428 & 210 & -741 & 234 & -835 & 226 & $\begin{array}{l}-989 \\
\end{array}$ & 244 \\
\hline-2429 & 210 & -742 & 234 & -844 & 228 & -990 & 244 \\
\hline-2430 & 178 & -743 & 235 & -845 & 228 & -991 & 243 \\
\hline-2431 & 178 & -744 & 235 & -846 & 228 & -999 & 243 \\
\hline-2432 & 178 & -745 & 235 & -847 & 229 & -1002 & 245 \\
\hline-2433 & 178 & -746 & 235 & -848 & 229 & -1003 & 245 \\
\hline-2412 & 176 & -747 & 235 & -862 & 228 & -1004 & 246 \\
\hline-2413 & 176 & -748 & 235 & -863 & 238 & & \\
\hline-2414 & 176 & -749 & 223 & -864 & 238 & SI & \\
\hline-2415 & 176 & -750 & 223 & -865 & 238 & -485 & 407 \\
\hline-2416 & 176 & -751 & 223 & -869 & 230 & -486 & 407 \\
\hline-2417 & 177 & -752 & 223 & -870 & 231 & -487 & 407 \\
\hline-2434 & 179 & -769 & 229 & -871 & 231 & -488 & 408 \\
\hline-2435 & 200 & -770 & 229 & -882 & 237 & -504 & 409 \\
\hline-2436 & 200 & -771 & 230 & -883 & 237 & -505 & 409 \\
\hline .2437 & 217 & -772 & 232 & -884 & 237 & -506 & 409 \\
\hline-2438 & 217 & -773 & 232 & -885 & 237 & -507 & 409 \\
\hline
\end{tabular}




\begin{tabular}{|c|c|c|c|c|c|c|c|}
\hline $\begin{array}{c}\text { Sample } \\
\text { no. }\end{array}$ & $\begin{array}{c}\text { Page } \\
\text { no. }\end{array}$ & $\begin{array}{c}\text { Sample } \\
\text { no. }\end{array}$ & $\begin{array}{c}\text { Page } \\
\text { no. }\end{array}$ & $\begin{array}{c}\text { Sample } \\
\text { no. }\end{array}$ & $\begin{array}{c}\text { Page } \\
\text { no. }\end{array}$ & $\begin{array}{c}\text { Sample } \\
\text { no. }\end{array}$ & $\begin{array}{c}\text { Page } \\
\text { no. }\end{array}$ \\
\hline SI & & SI & & $\mathrm{T}$ & & $\mathrm{T}$ & \\
\hline-508 & 409 & -680 & 402 & -771 & 436 & -842 & 443 \\
\hline-509 & 409 & -681 & 402 & -772 & 437 & -843 & 443 \\
\hline-530 & 405 & -682 & 402 & -774 & 442 & -844 & 443 \\
\hline-536 & 409 & -683 & 402 & -775 & 442 & -845 & 443 \\
\hline-537 & 409 & & & -776 & 443 & -846 & 423 \\
\hline-541 & 408 & SUA & & -777 & 442 & -848 & 438 \\
\hline-542 & 408 & -1 & 414 & -778 & 440 & -849 & 438 \\
\hline-544 & 408 & -6 & 414 & -779 & 440 & -850 & 438 \\
\hline-546 & 410 & -7 & 414 & -780 & 440 & -851 & 438 \\
\hline-547 & 410 & -8 & 414 & -781 & 439 & -852 & 421 \\
\hline-548 & 410 & -9 & 414 & -782 & 439 & -853 & 421 \\
\hline-549 & 410 & -11 & 415 & -783 & 439 & -854 & 428 \\
\hline-550 & 410 & -12 & 415 & -784 & 426 & -858 & 444 \\
\hline-551 & 411 & -13 & 414 & -785 & 426 & -859 & 444 \\
\hline-552 & 411 & -14 & 414 & -786 & 429 & -860 & 444 \\
\hline-.555 & 410 & -15 & 416 & -787 & 429 & -861 & 445 \\
\hline-556 & 410 & -16 & 416 & -788 & 441 & -866 & 423 \\
\hline-557 & 410 & -17 & 416 & -789 & 441 & -867 & 423 \\
\hline-576 & 402 & -18 & 416 & -790 & 422 & -868 & 423 \\
\hline-577 & 403 & -MS01 & 415 & -791 & 422 & -869 & 423 \\
\hline-578 & 401 & -MS02 & 415 & -792 & 422 & -879 & 441 \\
\hline-579 & 402 & & & -795 & 446 & -880 & 441 \\
\hline-580 & 402 & $T$ & & -796 & 420 & -881 & 444 \\
\hline-581 & 402 & -585 & 422 & -797 & 420 & -882 & 446 \\
\hline-609 & 403 & -626 & 440 & -801 & 432 & -883 & 446 \\
\hline-610 & 403 & -627 & 440 & -802 & 432 & -884 & 446 \\
\hline-617 & 405 & -628 & 441 & -803 & 432 & -885 & 447 \\
\hline-618 & 405 & -629 & 440 & -804 & 432 & -887 & 445 \\
\hline-619 & 405 & -663 & 422 & -805 & 433 & -888 & 445 \\
\hline-620 & 405 & -715 & 447 & -806 & 433 & -893 & 436 \\
\hline-621 & 405 & -726 & 426 & -807 & 433 & -891 & 437 \\
\hline-622 & 406 & -727 & 426 & -808 & 433 & -892 & 436 \\
\hline-623 & 406 & -728 & 426 & -809 & 433 & -893 & 437 \\
\hline-624 & 406 & -738 & 441 & -810 & 433 & -894 & 437 \\
\hline-625 & 406 & -739 & 441 & -811 & 433 & -895 & 437 \\
\hline-626 & 406 & -740 & 440 & -812 & 433 & -896 & 436 \\
\hline-627 & 406 & -741 & 441 & -813 & 434 & -897 & 436 \\
\hline-628 & 406 & -742 & 441 & -814 & 434 & -898 & 436 \\
\hline-629 & 406 & -744 & 430 & -815 & 434 & -899 & 437 \\
\hline-630 & 406 & -745 & 431 & -816 & 434 & -900 & 437 \\
\hline-631 & 406 & -746 & 431 & -817 & 434 & -905 & 435 \\
\hline-632 & 407 & -747 & 444 & -818 & 434 & -906 & 435 \\
\hline-633 & 407 & -748 & 421 & -819 & 435 & -910 & 431 \\
\hline-634 & 407 & -749 & 421 & -820 & 435 & $-915 A$ & 446 \\
\hline-635 & 407 & -750 & 423 & -821 & 431 & $-915 \mathrm{~B}$ & 447 \\
\hline-636 & 407 & -751 & 423 & -822 & 431 & -916 & 447 \\
\hline-637 & 408 & -752 & 423 & -823 & 431 & -921 & 420 \\
\hline-641 & 403 & -753 & 429 & -824 & 431 & -922 & 420 \\
\hline-642 & 403 & -754 & 429 & -825 & 432 & -923 & 420 \\
\hline-645 & 404 & -755 & 429 & -826 & 432 & -925 & 444 \\
\hline-646 & 404 & -756 & 428 & $-827 \mathrm{I}$ & 424 & -927 & 429 \\
\hline-647 & 404 & -757 & 429 & $-827 \mathrm{II}$ & 424 & -928 & 439 \\
\hline-648 & 404 & -758 & 442 & -828 & 424 & -929 & 439 \\
\hline-649 & 404 & -765 & 436 & -829 & 425 & -930 & 429 \\
\hline-650 & 404 & -766 & 436 & -830 & 425 & -936 & 430 \\
\hline$-65 \mathrm{IA}$ & 404 & -767 & 437 & -831 & 425 & -937 & 427 \\
\hline$-651 \mathrm{~B}$ & 404 & -768 & 438 & -839 & 422 & -938 & 427 \\
\hline-652 & 404 & -769 & 436 & -840 & 443 & -944 & 442 \\
\hline-679 & 402 & -770 & 436 & -841 & 443 & -951 & 448 \\
\hline
\end{tabular}




\begin{tabular}{|c|c|c|c|c|c|c|c|}
\hline $\begin{array}{c}\text { Sample } \\
\text { no. }\end{array}$ & $\begin{array}{c}\text { Page } \\
\text { no. }\end{array}$ & $\begin{array}{c}\text { Sample } \\
\text { no. }\end{array}$ & $\begin{array}{c}\text { Page } \\
\text { no. }\end{array}$ & $\begin{array}{c}\text { Sample } \\
\text { no. }\end{array}$ & $\begin{array}{c}\text { Page } \\
\text { no. }\end{array}$ & $\begin{array}{c}\text { Sample } \\
\text { no. }\end{array}$ & $\begin{array}{c}\text { Page } \\
\text { no. }\end{array}$ \\
\hline $\mathrm{T}$ & & TAM & & $\mathrm{Tx}$ & & $\mathrm{Tx}$ & \\
\hline-952 & 448 & -67 & 453 & -811 & 476 & -966 & 467 \\
\hline-953 & 1. 4449 & -68 & 453 & -812 & 476 & -967 & 467 \\
\hline$-954 \mathrm{~A}$ & $\therefore 449$ & -69 & 453 & -813 & 476 & -981 & 480 \\
\hline$-954 \mathrm{~B}$ & $\because 449$ & -70 & 453 & -814 & 476 & -982 & 480 \\
\hline-955 & $\quad 449$ & -72 & 453 & -815 & 477 & -983 & 481 \\
\hline-956 & $\quad 449$ & -73 & 454 & -816 & 477 & -984 & 481 \\
\hline-957 & 449 & -74 & 454 & -817 & 477 & -985 & 481 \\
\hline-958 & 449 & -75 & 453 & -818 & 478 & -986 & 481 \\
\hline-959 & 450 & -78 & 454 & -819 & 475 & -987 & 482 \\
\hline-960 & 450 & -79 & 453 & -820 & 477 & -988 & 482 \\
\hline-962 & 445 & -80 & 454 & -821 & 476 & -989 & 482 \\
\hline-963 & 429 & -81 & 454 & -822 & 477 & -990 & 482 \\
\hline-964 & 430 & -82 & 454 & $-823 \mathrm{~A}$ & 476 & -991 & 482 \\
\hline-965 & 430 & -85 & 454 & $-823 B$ & 476 & -992 & 481 \\
\hline-966 & 430 & -86 & 453 & -824 & 476 & -1026 & 483 \\
\hline-970 & 439 & -87 & 453 & -825 & 476 & -1027 & 483 \\
\hline-972 & 445 & -88 & 453 & -826 & 476 & -1028 & 483 \\
\hline-973 & 427 & -90 & 453 & -827 & 477 & -1029 & 483 \\
\hline-974 & 427 & -91 & 454 & -828 & 477 & -1030 & 483 \\
\hline-975 & 428 & -94 & 454 & -829 & 476 & -1031 & 483 \\
\hline-976 & 428 & -95 & 454 & -830 & 478 & -1032 & 483 \\
\hline-977 & 425 & -96 & 454 & -8.31 & 478 & -1033 & 483 \\
\hline-978 & 425 & -97 & 454 & -8.32 & 478 & -1034 & 483 \\
\hline-979 & 425 & -98 & 454 & -833 & 478 & -1035 & 483 \\
\hline-980 & 425 & -99 & 454 & -852 & 461 & -1071 & 470 \\
\hline-985 & 424 & -100 & 454 & -853 & 461 & -1072 & 471 \\
\hline-986 & 424 & -101 & 454 & -854 & 461 & -1073 & 471 \\
\hline-1006 & 447 & & & -8.56 & 478 & -1074 & 471 \\
\hline-1007 & 446 & TUNC & & -857 & 478 & -1078 & 463 \\
\hline-1008 & 447 & -1 & 457 & -8.58 & 478 & -1079 & 463 \\
\hline-1009 & 448 & -2 & 457 & -859 & 478 & -1080 & 463 \\
\hline-1010 & 448 & -3 & 458 & -860 & 478 & -1081 & 463 \\
\hline-1028 & 447 & -4 & 458 & -861 & 479 & -1082 & 464 \\
\hline-1045 & 446 & -5 & 458 & -862 & 479 & -1083 & 462 \\
\hline-1046 & 446 & -6 & 458 & -863 & 479 & -1084 & 462 \\
\hline-1100 & 421 & -7 & 458 & -864 & 479 & -1085 & 462 \\
\hline & & -8 & 458 & -865 & 479 & -1086 & 462 \\
\hline ТАМ & & -9 & 459 & -874 & 478 & -1087 & 462 \\
\hline-11 & 452 & -10 & 459 & -899 & 468 & -1089 & 464 \\
\hline-45 & 454 & -11 & 458 & -900 & 469 & -1090 & 464 \\
\hline-47 & 453 & -12 & 459 & -901 & 469 & -1091 & 462 \\
\hline-48 & 453 & & & -902 & 469 & -1092 & 464 \\
\hline-49 & 453 & $\mathrm{Tx}$ & & -928 & 472 & -1093 & 464 \\
\hline-50 & 453 & -577 & 473 & -929 & 472 & -1094 & 464 \\
\hline-51 & 453 & -578 & 473 & -930 & 472 & -1095 & 464 \\
\hline-53 & 453 & -579 & 473 & -931 & 472 & -1096 & 464 \\
\hline-.54 & 453 & -580 & 474 & -932 & 472 & -1097 & 464 \\
\hline-55 & 453 & -581 & 473 & -954 & 468 & -1098 & 465 \\
\hline-56 & 453 & -582 & 473 & -955 & 467 & -1099 & 465 \\
\hline-.57 & 453 & -583 & 474 & -956 & 466 & -1100 & 462 \\
\hline-58 & 453 & -584 & 474 & -957 & 466 & -1101 & 465 \\
\hline-59 & 453 & -585 & $47 \cdot 1$ & -958 & 468 & -1102 & 465 \\
\hline-60 & 453 & -694 & 474 & -959 & 468 & -1103 & 463 \\
\hline-61 & 453 & $-694 A$ & 474 & -960 & 467 & -1104 & 465 \\
\hline-62 & 453 & -695 & 474 & -961 & 467 & -1105 & 465 \\
\hline-63 & 453 & -807 & 475 & -962 & 467 & -1106 & 465 \\
\hline-64 & 453 & -808 & 475 & -963 & 467 & -1107 & 465 \\
\hline-65 & 453 & -809 & 475 & -964 & 467 & -1108 & 465 \\
\hline-66 & 453 & -810 & 475 & -965 & 466 & -1109 & 465 \\
\hline
\end{tabular}




\begin{tabular}{|c|c|c|c|c|c|c|c|}
\hline $\begin{array}{c}\text { Sample } \\
\text { no. }\end{array}$ & $\begin{array}{c}\text { Page } \\
\text { no. }\end{array}$ & $\begin{array}{c}\text { Sample } \\
\text { no. }\end{array}$ & $\begin{array}{c}\text { Page } \\
\text { no. }\end{array}$ & $\begin{array}{c}\text { Sample } \\
\text { no. }\end{array}$ & $\begin{array}{c}\text { Page } \\
\text { no. }\end{array}$ & $\begin{array}{c}\text { Sample } \\
\text { no. }\end{array}$ & $\begin{array}{c}\text { Page } \\
\text { no. }\end{array}$ \\
\hline $\mathbf{T x}$ & & $\mathrm{U}$ & & $\mathbf{U}$ & & UGa & \\
\hline-1110 & 466 & -746 & 265 & -2194 & 263 & -109 & 489 \\
\hline-1111 & 463 & -747 & 265 & -2195 & 263 & -110 & 490 \\
\hline-1112 & 463 & -750 & 255 & -2196 & 263 & -111 & 490 \\
\hline-1118 & 463 & -751 & 257 & -2197 & 263 & -117 & 488 \\
\hline-1119 & 484 & -752 & 266 & -2198 & 263 & -121 & 487 \\
\hline-1121 & 484 & -753 & 264 & -2199 & 264 & -123 & 488 \\
\hline-1122 & 484 & -754 & 269 & -2200 & 264 & -126 & 487 \\
\hline-1123 & 484 & -755 & 269 & -2201 & 264 & -127 & 487 \\
\hline-1124 & 485 & -756 & 269 & -2202 & 264 & -128 & 488 \\
\hline-1157 & 469 & -2056 & 261 & -2203 & 264 & -129 & 494 \\
\hline-1158 & 469 & -2132 & 258 & -2204 & 266 & -132 & 488 \\
\hline-1159 & 469 & -2133 & 257 & -2205 & 259 & -133 & 486 \\
\hline-1160 & 469 & -2135 & 250 & -2206 & 259 & -136 & 488 \\
\hline-1161 & 469 & -2136 & 250 & -2207 & 260 & -137 & 487 \\
\hline-1162 & 469 & -2137 & 250 & -2219 & 265 & -138 & 487 \\
\hline-1163 & 469 & -2138 & 250 & -2220 & 265 & -140 & 494 \\
\hline-1164 & 470 & -2139 & 249 & -2221 & 265 & -145 & 495 \\
\hline-1165 & 470 & -2140 & 249 & -2222 & 265 & -146 & 492 \\
\hline-1166 & 470 & -2141 & 249 & -2223 & 266 & -147 & 495 \\
\hline-1218 & 471 & -2142 & 250 & -2224 & 255 & -148 & 495 \\
\hline-1219 & 471 & -2143 & 249 & -2225 & 254 & -149 & 492 \\
\hline-1220 & 471 & -2144 & 249 & -2226 & 254 & -150 & 499 \\
\hline-1273 & 480 & -2145 & 249 & -2227 & 255 & -151 & 492 \\
\hline-1275 & 479 & -2146 & 254 & -2228 & 256 & -152 & 492 \\
\hline-1276 & 479 & -2147 & 254 & -2229 & 256 & -153 & 488 \\
\hline-1279 & 480 & -2148 & 250 & -2261 & 266 & -154 & 487 \\
\hline-1280 & 480 & -2149 & 250 & -2270 & 256 & -155 & 487 \\
\hline & & -2150 & 251 & -2271 & 256 & -156 & 488 \\
\hline $\mathrm{U}$ & & -2151 & 251 & -2272 & 256 & -158 & 493 \\
\hline-169 & 257 & -2152 & 251 & -2273 & 257 & -159 & 493 \\
\hline-500 & 260 & -2153 & 2.51 & -2274 & 257 & -160 & 487 \\
\hline-600 & 261 & -2155 & 251 & -2275 & 257 & -161 & $49 !$ \\
\hline-601 & 261 & -2156 & 251 & -2276 & 259 & -162 & 488 \\
\hline-602 & 261 & -2157 & 252 & -2278 & 269 & -163 & 493 \\
\hline-612 & 261 & -2158 & 252 & -2279 & 269 & -164 & 493 \\
\hline-689 & 268 & -2159 & 252 & -2280 & 269 & -165 & 488 \\
\hline-690 & 268 & -2160 & 252 & -2281 & 267 & -166 & 493 \\
\hline-691 & 268 & -2161 & 252 & -2282 & 267 & -167 & 493 \\
\hline-692 & 268 & -2162 & 252 & -2283 & 267 & -169 & 487 \\
\hline-693 & 268 & -2163 & 253 & -2284 & 267 & -170 & 491 \\
\hline-694 & 268 & -2164 & 253 & -2285 & 267 & -171 & 487 \\
\hline-705 & 265 & -2165 & 253 & & & -172 & 491 \\
\hline-706 & 265 & -2166 & 253 & UGa & & -173 & 491 \\
\hline-707 & 259 & -2167 & 255 & -79 & 491 & -174 & 491 \\
\hline-708 & 260 & -2168 & 267 & -80 & 491 & -175 & 488 \\
\hline-709 & 260 & -2169 & 267 & -87 & 489 & $-176 \mathrm{~A}$ & 495 \\
\hline-710 & 260 & -2170 & 267 & -88 & 489 & $-176 \mathrm{~B}$ & 495 \\
\hline-713 & 258 & -2171 & 267 & -90 & 489 & -177 & 487 \\
\hline-714 & 258 & -2172 & 267 & -91 & 486 & -178 & 488 \\
\hline-715 & 258 & -2184 & 262 & -93 & 489 & -179 & 495 \\
\hline-716 & 258 & -2185 & 262 & -95 & 489 & -180 & 488 \\
\hline-717 & 259 & -2186 & 262 & -97 & 489 & -184 & 487 \\
\hline-718 & 259 & -2187 & 262 & -98 & 489 & -187 & 486 \\
\hline-719 & 259 & -2188 & 262 & -99 & 489 & -188 & 486 \\
\hline-724 & 254 & -2189 & 262 & -100 & 490 & -189 & 488 \\
\hline-725 & 254 & -2190 & 262 & -102 & 490 & -190 & 496 \\
\hline-726 & 255 & -2191 & 263 & -103 & 490 & -191 & 496 \\
\hline-727 & 256 & -2192 & 263 & -106 & 487 & -192 & 496 \\
\hline-743 & 267 & -2193 & 263 & -107 & 489 & -193 & 496 \\
\hline
\end{tabular}




\begin{tabular}{cccccccc}
\hline $\begin{array}{c}\text { Sample } \\
\text { no. }\end{array}$ & $\begin{array}{c}\text { Page } \\
\text { no. }\end{array}$ & $\begin{array}{c}\text { Sample } \\
\text { no. }\end{array}$ & $\begin{array}{c}\text { Page } \\
\text { no. }\end{array}$ & $\begin{array}{c}\text { Sample } \\
\text { no. }\end{array}$ & $\begin{array}{c}\text { Page } \\
\text { no. }\end{array}$ & $\begin{array}{c}\text { Sample } \\
\text { no. }\end{array}$ & $\begin{array}{c}\text { Page } \\
\text { no. }\end{array}$ \\
\hline UGa & & UGa & & VRI & & VRI & \\
-194 & 488 & -223 & 496 & -188 & 498 & -207 & 503 \\
-195 & 486 & -225 & 494 & -189 & 498 & -209 & 499 \\
-196 & 496 & -226 & 494 & -191 & 503 & -210 & 499 \\
-198 & 497 & -227 & 495 & -192 & 500 & -211 & 499 \\
-199 & 495 & -229 & 491 & $-193 / 1$ & 500 & -212 & 501 \\
-202 & 487 & -230 & 490 & $-193 / 2$ & 500 & -213 & 501 \\
-207 & 486 & -231 & 490 & -194 & 500 & -214 & 501 \\
-209 & 487 & -232 & 490 & -196 & 504 & -215 & 501 \\
-211 & 488 & -243 & 496 & -197 & 504 & -223 & 499 \\
-212 & 493 & -244 & 496 & -198 & 498 & -224 & 499 \\
-214 & 492 & & & -199 & 500 & -225 & 499 \\
-216 & 487 & VRI & & -201 & 503 & -226 & 500 \\
-217 & 492 & -174 & 504 & -202 & 504 & -229 & 502 \\
-218 & 491 & -183 & 503 & -203 & 504 & -230 & 501 \\
-219 & 491 & -184 & 504 & -204 & 501 & -243 & 502 \\
-221 & 492 & -185 & 503 & -205 & 500 & -244 & 502 \\
-222 & 492 & -187 & 498 & -206 & 499 & -248 & 502
\end{tabular}

